\title{
HYBRID CANONICAL CORRELATION ANALYSIS AND REGRESSION FOR RADIOMETRIC NORMALIZATION OF CROSS-SENSOR SATELLITE IMAGES
}

\author{
L. G. Denaro ${ }^{1}$, C. H. Lin $^{1}$ \\ ${ }^{1}$ National Cheng Kung University (NCKU), Tainan - Taiwan
}

Commission IV

KEY WORDS: Relative radiometric normalization, pseudo-invariant features, kernel canonical correlation analysis, nonlinear regression.

\begin{abstract}
:
Relative radiometric normalization (RRN) minimizes radiometric differences among images caused by inconsistencies of acquisition condition. In this study, a cross-sensor RRN method is proposed for optical satellite images from Landsat 8 OLI (L8) and Landsat 7 ETM+ (L7) sensors. The data from these two sensors have different pixel depths. Therefore, a rescaling on the radiometry resolution is performed in the preprocessing. Then, multivariate alteration detection (MAD) based on kernel canonical correlation analysis (KCCA) is adopted, which is called KCCA-based MAD, to select pseudo-invariant features (PIFs). The process of RRN is performed by using polynomial regression with Gaussian weighted regression. In experiments, qualitative and quantitative analyses on images from different sensors are conducted. The experimental result demonstrates the superiority of the proposed nonlinear transformation, in terms of regression quality and radiometric consistency, compared with RRN using linear regression.
\end{abstract}

\section{INTRODUCTION}

Remote sensing based on satellite images is one of the techniques to analyze the earth spatial condition. Landsat program is the one having the longest history [1]. Landsat 1 was launched in 1972, and the last generation is Landsat 8 which was launched in 2013 and is currently still operating [2]. The long history of Landsat data is one of the most valuable datasets available for studying land cover change and human influences on the land surface, especially since the first Thematic Mapper (TM) sensor was launched in 1982, which provided higher spatial resolution and more spectral bands [3].

For this long-term data provision, we can use this advantage for many purposes of research. Consequently, we will retrieve the gaining of the newest sensor but in contrast with the old sensors [4]. The advantages of the newest Landsat satellites would make the observation is become easier since using advanced technologies. However, the data of the old Landsat may have some lacks in storing procedure or in the sensor itself. Moreover, instead of those problem, spectral data acquired by satellite sensors are influenced by a number of factors, such as atmospheric absorption and scattering, sensor target illumination geometry, and image data processing procedure, which tend to change through times [5]. Targets in different sensors and acquisition date may have many variables such as different pixel properties, sensor advanced generation etc. In order to detect genuine landscape changes as revealed by changes in surface reflectance from multi-date satellite images, it is necessary to carry out radiometric normalization [5].

Due to different spectral signatures, nonlinearly approach is required rather than linear approach done by [6][7][8]. Recent research conducted by [1][9][10][11], the PIFs selection is done by the nonlinearity that use projection of the data to higher dimensional feature space called Kernel function. Therefore, this method is called as kernel multivariate alteration detection (KMAD). KMAD maintains the PIFs by projecting the samples into the higher dimensional feature space and assume it as nonlinearity from the original feature space. Furthermore, to complete this study, nonlinear regression applied with Gaussian distribution function is preferred to generate relative radiometric normalization.

\section{METHODOLOGY}

\subsection{Pseudo-invariant Feature Selection}

In order to retrieve the invariant pixels between images in difference dates, call date 1 and date 2 in bitemporal image, we suppose to form the integration between them to find optimum correlation to discriminate pseudo invariant features (PIFs). There are many methods to extract PIFs, in this paper we would like to Kernel Canonical Correlation Analysis with the use of Multivariate Alteration Selection (MAD) method to change detection. In case of using the data, in this paper we provide date 1 represented as $\mathrm{X}$ and date 2 represented as Y of Landsat Imagery namely Landsat 7 and Landsat 8 respectively. Image $X$ and image $Y$ have $p$ and $q$ of bands respectively and each band has the same $n$ pixel numbers. To make the matrices, we assume $\mathrm{X}$ and $\mathrm{Y}$ are pair of multiple vectors. Therefore, $\mathrm{X}$ and $\mathrm{Y}$ have numbers of vector bands respectively as shown below.

$$
\begin{aligned}
& X_{p x n}=\left[\begin{array}{lllllllllllllll}
X_{11} & X_{11} & \ldots & X_{1 n} & X_{21} & X_{21} & \ldots & X_{2 n} & \ldots & X_{p 1} & \ldots & X_{p 2} & \ldots & \ldots & \ldots \\
X_{p n}
\end{array}\right]
\end{aligned}
$$

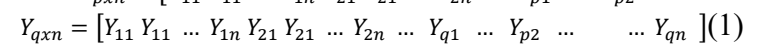

Then, require the mean of each rows of $\mathbf{X}$ and $\mathbf{Y}$ images vectors called $\mathrm{E}\left(X_{p}\right)$ and $\mathrm{E}\left(Y_{q}\right)$ respectively. From the both mean variables mentioned, we retrieve the variance and covariance matrix vectors and generate correlation function subject to its variance (2). 


$$
\begin{aligned}
& \rho=\frac{\operatorname{Cov}(U, V)}{\sqrt{\operatorname{Var}(U) \times \operatorname{Var}(V)}}=\frac{a^{T} \sum_{X Y} b}{\sqrt{a^{T} \sum_{X X} a b^{T} \sum_{Y Y} b}} \rho=\operatorname{Corr}(U, V)= \\
& \frac{\operatorname{Cov}(U, V)}{\sqrt{\operatorname{Var}(U) x \operatorname{Var}(V)}}=\frac{a^{T} \sum_{X Y} b}{\sqrt{a^{T} \sum_{X X} a b^{T} \sum_{Y Y} b}} \rho=\operatorname{Corr}(U, V)=\frac{\operatorname{Cov}(U, V)}{\sqrt{\operatorname{Var}(U) x \operatorname{Var}(V)}}= \\
& \frac{a^{T} \sum_{X Y} b}{\sqrt{a^{T} \sum_{X X} a b^{T} \sum_{Y Y} b}}
\end{aligned}
$$

By using Lagrange multiplier, this leads to the set of eigenvalues and eigenvector. In terms of higher dimensional feature space, the argument from Eq. (2) is then transformed to,

$\arg \operatorname{Corr}\left(\alpha^{T} K_{x}, \beta^{T} K_{y}\right)=\frac{\alpha^{T} K_{x} K_{y} \beta}{\sqrt{\alpha^{T} K_{x} K_{x} \alpha \beta^{T} K_{y} K_{y} \beta}}$

where $a$ and $b$ are replaced by $\varphi(X) \times \alpha$ and $\varphi(X) \times \beta$ with the mapping function $\varphi$ and $K_{x}=\varphi\left(X^{T}\right) \varphi(X)$ and $K_{y}=\varphi\left(Y^{T}\right) \varphi(Y)$. The more detailed equation, the readers are suggested to read kernel canonical correlation analysis [1].

Therefore, according to [1], the PIFs selection is done by normalization on MAD in higher dimension denoted by,

$N M A D=\sum_{i=1}^{p}\left(\frac{M A D_{i}}{\rho_{M A D_{i}}}\right)^{2}<t$,

where the decision threshold $(t)$ is based on chi-square test. this threshold defines that if the values are smaller, the values could be called as no-change or PIFs. Subsequently, the sum of the mad square divided by standard deviation square refers to the degree of freedom $p$.

The algorithm discussed before is KCCA-based MAD method that is the basic concept of change detection done in the higher dimensional feature space. This method deals with the nonlinear assumption of the reflectance and heterogeneity.

\subsection{Linear and nonlinear regression}

To define the parameters that generate the normalized image, the key is to use selectable or acceptable PIFs. These selected PIFs have been done in the previous chapter. The least square regression is used to develop fitting line between two images, PIFs of Landsat 7 and Landsat 8 . The two methods use polynomial regression from degree 1 to degree 5 as shown in Figure 1. The linear regression is stated in Figure 3 for degree 1. We can see that there are having particular characteristics of fitting line motion or curve. For the polynomial regression, the curve-lines are problematic in the mapping function to $y$-value axis. For example, the problematic mapping functions appear merely visible to degree 3 to degree 5 . However, the degree 2 also involves in the higher gray level values. Therefore, only linear fitting line is acceptable to conduct mapping function.

Therefore, this strategy to transform the digital image tied up to the reference is believable and become major discussion with recombination to Gaussian distribution function as proposed method.

This Gaussian function is set to be weighting that bound to linear and nonlinear function. There is a condition to determine the standard deviation $\sigma$ and mean $\mu$ as explained in equation (5). $x$ is gray value digital number.

$$
f(x, \sigma, \mu)=e^{\frac{-(x-\mu)^{2}}{2 \sigma^{2}}}
$$

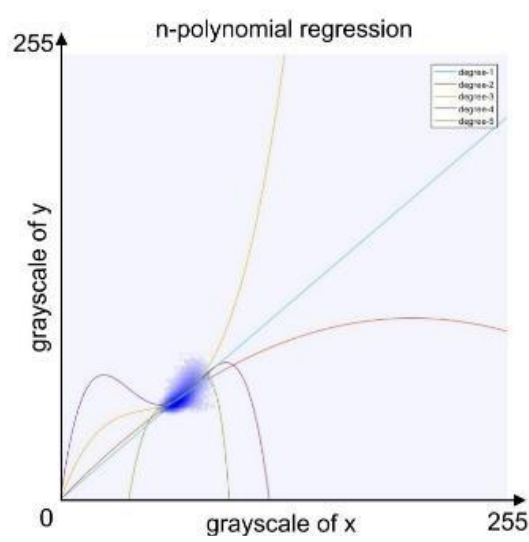

Figure 1. Polynomial least square regression

To better understanding in equation (5), it can be clearly depicted in Figure 2 about how to determine the Gaussian weighting distribution function.

\section{Gaussian weighting distribution}

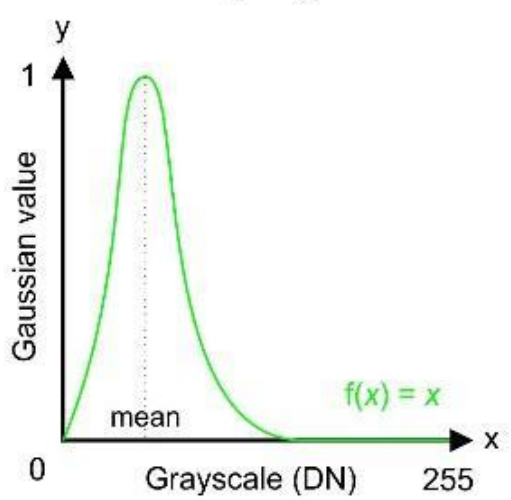

Figure 2. Proposed Gaussian weighting function

\subsection{Normalization technique}

Normalization technique is the last step of image transformation. The image that needs to be transformed is at first treated as dependent variable. In this case, Landsat 8 imagery is going to be transformed to Landsat 7 radiometric level (common level) by using equation (6)

$\underline{Y}=f(x, \sigma, \mu) * R_{1}(x)+(1-f(x, \sigma, \mu)) * R_{2}(x)$

$R_{1}$ and $R_{2}$ are the linear (degree-1) and nonlinear (degree-2) of regression function.

\section{STUDY AREA}

Landsat data of Taiwan and Japan region for 2013 to 2017 were used in this research. There are two datasets at different sensor of each location, namely Landsat 7 ETM+ and Landsat 8 OLI. Landsat 7 images were selected as the reference image. 


\section{RESULT AND DISCUSSION}

This section describes the visual and statistical result from three different RRN methods. The visual result is determined by comparing of normalized data images by computer visualization to overall performance of the methods. Both of normalized images and reference images are displayed on the monitor screen, and the visual closeness of each normalized image to the reference image is determined by qualitatively. If the visual image changes approaching the reference and become similar or identical, the image can be regarded as radiometric adjusted to the reference image as shown in Figure 6. However, this visual comparison method has less convinced since our visual is prone to subjectivity. Therefore, a quantitative comparison is preferable.
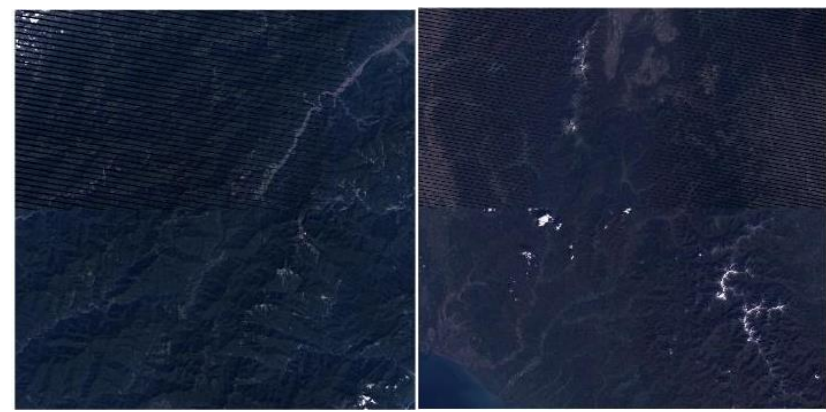

Figure 3. Mosaic images of Landsat 7 (up) and Landsat 8 (down) of normalized images. Taiwan (left), Japan (right).

\subsection{Root mean square error (RMSE)}

In quantitative comparison, RMSE is applied. RMS error is used to measure the statistical agreement of normalized image with the reference image.

\begin{tabular}{|c|c|c|c|c|c|c|}
\hline \multirow{2}{*}{ Band } & \multicolumn{3}{|c|}{ Taiwan (RMSE) } & \multicolumn{3}{c|}{ Japan (RMSE) } \\
\cline { 2 - 7 } & LN & NL & PM & LN & NL & PM \\
\hline 1 & 1.23 & 1.26 & $\mathbf{1 . 2 6}$ & 1.82 & 1.80 & $\mathbf{1 . 8 0}$ \\
\hline 2 & 1.08 & 1.16 & $\mathbf{1 . 1 6}$ & 2.03 & 2.03 & $\mathbf{2 . 0 0}$ \\
\hline 3 & 1.29 & 1.42 & $\mathbf{1 . 4 1}$ & 2.33 & 2.36 & $\mathbf{2 . 3 5}$ \\
\hline 4 & 2.29 & 7.02 & $\mathbf{6 . 6 4}$ & 4.07 & 6.65 & $\mathbf{6 . 2 5}$ \\
\hline 5 & 2.34 & 6.69 & $\mathbf{6 . 4 1}$ & 3.32 & 6.11 & $\mathbf{6 . 0 0}$ \\
\hline 6 & 1.48 & 2.89 & $\mathbf{2 . 8}$ & 2.33 & 3.38 & $\mathbf{3 . 3 3}$ \\
\hline
\end{tabular}

Table 1. Normalized images: Root mean square error (RMSE)

You can see the algorithm in the Eq. (7) as follows.

$R M S E_{p}=\sqrt{\frac{\sum_{1}^{n}\left(D N_{e s t}^{\prime}-D N_{r e f}\right)^{2}}{n}}$,

where $D N_{e s t}^{\prime}$ represents the normalized digital number of band $p$ of Landsat 8 imagery, $D N_{r e f}$ is the digital number of band $\mathrm{q}$ in reference image. Thus, the digital number of pixels of the normalized image are compared with different radiometric normalization. If the difference between these numbers is quite small, the RMSE result will be small, this is implying that the Landsat 8 imagery is radiometrically corrected to the reference image. The result of RMSE as follows on Table 1.

\section{CONCLUSION}

This study presents a KCCA-based MAD for the PIFs extraction and Gaussian weighting regression for normalization on the Landsat 7 and Landsat 8 imagery. The PIFs extraction method is tried to select acceptable invariant feature of bitemporal image nonlinearity. To treat the heterogeneous pixel signatures, the proposed normalization procedure is then applied. This kind of procedure become more flexible in relative radiometric normalization.

\section{REFERENCES}

[1] L. G. Denaro, L. Bo-Yi, M. A. Syariz, L. M. Jaelani, and C.H. Lin, "Pseudoinvariant feature selection for cross-sensor optical satellite images," J. Appl. Remote Sens., vol. 11, no. 1, 2018.

[2] N. Mishra, M. O. Haque, L. Leigh, D. Aaron, D. Helder, and B. Markham, "Radiometric cross calibration of landsat 8 Operational Land Imager (OLI) and landsat 7 enhanced thematic mapper plus (ETM+)," Remote Sens., vol. 6, no. 12, pp. 1261912638, 2014.

[3] Z. Zhu and C. E. Woodcock, "Object-based cloud and cloud shadow detection in Landsat imagery," Remote Sens. Environ., vol. 118, pp. 83-94, 2012.

[4] B. L. Markham and D. L. Helder, "Forty-year calibrated record of earth-reflected radiance from Landsat: A review," Remote Sens. Environ., vol. 122, pp. 30-40, 2012.

[5] X. Yang and C. P. Lo, "Relative radiometric normalization performance for change detection from multi-date satellite images," Photogramm. Eng. Remote Sensing, vol. 66, no. August, pp. 967980, 2000.

[6] M. A. Syariz, B. Y. Lin, L. G. Denaro, L. M. Jaelani, M. Van Nguyen, and C. H. Lin, "Spectral-consistent relative radiometric normalization for multitemporal Landsat 8 imagery," ISPRS $J$. Photogramm. Remote Sens., vol. 147, no. March 2018, pp. 56-64, 2019.

[7] M. J. Canty and A. A. Nielsen, "Automatic radiometric normalization of multitemporal satellite imagery with the iteratively re-weighted MAD transformation," Remote Sens. Environ., vol. 112, no. 3, pp. 1025-1036, 2008.

[8] C.-H. Lin, B.-Y. Lin, K.-Y. Lee, and Y.-C. Chen, "Radiometric normalization and cloud detection of optical satellite images using invariant pixels," ISPRS J. Photogramm. Remote Sens., vol. 106, pp. 107-117, 2015.

[9] D. Hardoon, S. Szedmak, and J. Shawe-Taylor, "Canonical Correlation Analysis: An Overview with Application to Learning Methods," Neural Comput., vol. 16, no. 12, pp. 2639-2664, 2004.

[10] M. Volpi, G. Camps-Valls, and D. Tuia, "Spectral alignment of multi-temporal cross-sensor images with automated kernel canonical correlation analysis," ISPRS J. Photogramm. Remote Sens., vol. 107, pp. 50-63, 2015.

[11] Y. Bai, P. Tang, and C. Hu, "Kernel Mad Algorithm for Relative Radiometric Normalization," ISPRS Ann. Photogramm. Remote Sens. Spat. Inf. Sci., vol. III-1, no. July, pp. 49-53, 2016. 